\title{
Prevalência de hipocalcemia subclínica em vacas leiteiras no período de transição no sertão da Paraíba
}

Beatriz Dantas Fernandes;, Jéssica Monique dos Santos Lima, Daniel Cézar da Silva, Gilderlândio Pinheiro Rodrigues, Déborah Lanne Barros Dias, Bismark Alves da Silva

Instituto Federal da Paraíba (IFPB), Sousa, PB, Brasil

*Autor correspondente

e-mail: beatriz_dfernandes@hotmail.com

\section{Resumo}

A hipocalcemia subclínca é uma desordem metabólica ocasionada pela diminuição dos níveis séricos de cálcio (Ca) no sangue, ocorrendo frequentemente e podendo acometer até $50 \%$ dos animais do rebanho. Este distúrbio acontece em decorrência da mobilização deste macroelemento mineral pelo metabolismo da vaca leiteira no período do pré e pós-parto, sem manifestações clínicas evidentes, ocasionando diminuição na produção de leite e causando grandes prejuízos na bovinocultura leiteira. No período de pré e pós-parto se verifica o maior incremento nas exigências de cálcio pelo organismo da fêmea em lactação, e a demanda repentina deste macromineral pode levar a diversos distúrbios, como redução na ingestão de matéria seca, menor desempenho reprodutivo, retenção de placenta, mastite, metrite, entre outras doenças metabólicas e infecciosas. 0 presente trabalho foi realizado no período de abril a dezembro de 2016 com o objetivo de avaliar a prevalência de hipocalcemia subclínica em vacas leiteiras que se encontravam em período de transição (pré e pós-parto), inseridas em propriedades na microrregião de Sousa e município de São João do Rio do Peixe, ambos no estado da Paraíba. Foram coletadas 456 amostras de sangue, as quais foram encaminhadas ao Laboratório de Análises Clínicas do Hospital Veterinário do Instituto Federal da Paraíba/Campus Sousa para posterior análise. Os tubos foram centrifugados a $3.000 \mathrm{rpm}$ por 15 minutos, retirando-se o soro, no qual foi determinado concentração de Ca total e ionizado pela técnica de colorimetria, utilizando-se kit bioquímico Cálcio Doles. Na avaliação dos níveis séricos de Ca total em função do período reprodutivo das vacas, verificou-se que 37,42 e 41,86\% das vacas no período de pré e pós-parto, respectivamente, estavam abaixo do limite inferior $(7,9 \mathrm{mg} / \mathrm{dL})$, ou seja, encontravam-se em um quadro de hipocalcemia subclínica. Para os níveis séricos de Ca ionizado verificou-se menor percentual de animais em condição de hipocalcemia subclínica. Nesta situação, registra-se que 21,29\% das vacas no pré-parto e 20,93\% das vacas no pós-parto estavam abaixo do limite inferior $(3,79 \mathrm{mg} / \mathrm{dL})$. Não houve diferença significativa $(\mathrm{P}>0,05)$ para os níveis 
séricos de Ca total e ionizado em função da situação reprodutiva das vacas, indicando a condição de pré e pós-parto não ocasionou alterações dos parâmetros avaliados. A carência de pastagens de boa qualidade somada à suplementação mineral inadequada para as vacas e, principalmente, a avaliação bioquímica de Ca são as principais informações para diagnóstico de hipocalcemia subclínica. Este foi o primeiro trabalho realizado na região para avaliação da prevalência de hipocalcemia subclínica, ressaltando a importância e necessidade de ações de caráter informativo aos produtores sobre o fornecimento adequado de suplemento mineral, considerada estratégia eficaz para redução de vacas acometidas pelo distúrbio. 